\title{
Brigitte Hasenjürgen und Martin Spetsmann-Kunkel (2019). Kulturalisierungsprozesse in Bildungskontexten. Bildungsaspiration von Jugendlichen aus Südosteuropa
}

\author{
Baden-Baden: Nomos, ISBN 978-3-8487-4926-3 (Print), ISBN 978-3-8452-9139-0 \\ (ePDF), 222 S., $44 €$
}

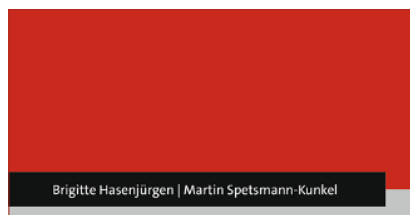

Kulturalisierungsprozesse in Bildungskontexten

C Nomos $\sum$
Der von Brigitte Hasenjürgen und Martin Spetsmann-Kunkel herausgegebene Band versammelt verschiedene Perspektiven auf das Forschungsprojekt zu Selbst- und Fremdeinschätzungen der Bildungsaspiration von neuzugewanderten Jugendlichen aus den EU-Ländern Bulgarien, Rumänien, Kosovo und Serbien, die in zehn Beiträgen von verschiedenen Autor_innen bearbeitet und über zwei Gastbeiträge ergänzt werden. Die Besonderheit des Bandes besteht in der theoretisch-empirischen Reflexion des „Mythos von bildungsdesinteressierten, als „Roma“ markierten Migrant"innen aus Südosteuropa " und die darüber ermöglichten Einblicke in thematische Schwerpunktsetzungen, in denen Perspektiven verschiedener Akteursgruppen zur Geltung kommen: Das Erfahrungsspektrum von Jugendlichen, ebenso wie berufliche Routinen und Sichtweisen von Lehrer_innen und Sozialarbeiter_innen. Hervorzuheben ist zudem der Einblick in das methodische Vorgehen (Spetsmann-Kunkel) und die forschungspraktische Reflexion im Hinblick auf die Anlage einer rassismuskritischen Forschung im Vortrag (Wienand) und im Hinblick auf eine dolmetschergestützte Interviewdurchführung (Gumenik \& Quasinowski).

Den einleitenden Bemerkungen der Herausgeber_innen folgt zunächst der Beitrag von Hasenjürgen zur Konturierung des Forschungsrahmens. Die Analyse von
Kulturalisierungen in Bildungsprozessen wird in dreierlei Hinsicht in den Fokus von Bildungsungleichheiten gerückt und theoretisierend kontextualisiert: Mit Bezügen zu Werkzeugen von Bourdieu wird auf soziale Effekte des Bildungssystems hingewiesen, die Perspektive Doing migration aufgegriffen und der migrationsund rassismuskritische Zugang, wie auch mithilfe des Capability Approach das Recht auf Schule und Ausbildung hervorgehoben. Hasenjürgen bietet zudem einen Überblick zu empirischen Befunden, mit denen sie den stabilen Zusammenhang von sozialer Herkunft und Bildung, migrationsspezifische Diskriminierungseffekte und die Bildungssituation von Jugendlichen aus RomGruppen diskutiert. Über diese theoretisch-empirische Rahmung konturiert Hasenjürgen den Forschungsrahmen, für den neben der Relevanz der Frageformen, etwa auf die Notwendigkeit einer Forschungssensibilität gegenüber als „fremd“ markierten Interviewpartner_innen und die Wahrnehmung von Rassismuserfahrungen hingewiesen wird.

In dem anschließenden Beitrag von Hasenjürgen/Quasinowski werden die 48 von 60 interviewten Jugendlichen als eine heterogene Gruppe beschrieben. Fokussiert werden daran anknüpfend drei Portraits von Jugendlichen, die ihre vorgestellten Bildungserfahrungen konturieren und vor dem Hintergrund „tatsächlicher und vorgestellter" Familie analysiert werden. Bedeutsam dabei sind zum einen die Ergebnisse, die einer Etikettierung der Jugendlichen als ausschließliche „Familienmenschen “ widersprechen, auf einen potentialorientierten Blick auf die Eltern hinweisen und auf die Unterstützung der Familie bei alltäglichen Diskriminierungen aufmerksam machen. Zum anderen bietet der Beitrag eine anregende Reflexion der Normalitätsvor- 
stellungen von Familie und der damit zusammenhängenden Zuschreibungen von pädagogischen Fachkräften bei Adressierungen von jugendlichen Roma und Romnija.

Wienand hingegen widmet sich in ihrem Beitrag der Begabungsrhetorik am Beispiel der Berufsorientierung, in dem kontrastiv die Perspektiven einer Mitarbeiterin aus einer berufsvorbereitenden Maßnahme im handwerklichen Bereich und eines Jugendlichen beleuchtet werden. Hervorzuheben ist der Fokus auf die Rede von Begabung, die als „Fremdzuschreibung einer Begabung durch Akteur*innen [...] an Jugendliche als individualisierte Erklärung für pädagogische Beurteilungen “ und als "Selbstzuschreibung einer Begabung von Jugendlichen" als Begründungsmotiv des eigenen Bildungspfads herangezogen werden kann. Während Wienand für die pädagogische Perspektive institutionelle Differenzordnungen in den analytischen Blick nimmt, wird die Perspektive des Jugendlichen im Rahmen von Agency betrachtet. Als Pendant zu dieser individualisierenden Argumentationsstruktur können Otheringsprozesse in der pädagogischen Arbeit festgestellt werden, die Spetsmann-Kunkel in seinem Beitrag in den Mittelpunkt rückt. Er diskutiert fünf der insgesamt 15 detailliert analysierten Interviews mit professionellen Fachkräften, der er eine theoretische Auseinandersetzung mit Ethnisierung, Kulturalisierung und dem Zusammenhang von kategorialen Klassifikationen und Rassismus voranstellt, sowie die diskursive und strukturelle Position der Rom-Gruppen bespricht.

Zwei weitere empirisch fundierte Beiträge von Hasenjürgen gehen auf das Modell Hauptschule und die Bildungssituation von eingewanderten Jugendlichen ein. Hasenjürgen zeichnet in der Analyse der Interviews von drei Schulleiter_innen Strategien der Bestandssicherung der sich in Auflösung befindenden Hauptschulen nach, für die sie eine Instrumentalisierung ,ausländischer Schüler_innen“ herausarbeiten kann, die zugleich mit dem Anspruch eben diese zu „beschützen“ einhergeht. In dem zweiten Beitrag steht die „Praxis der Verweisung älterer Quereinsteiger*innen aus dem Balkan “von der Regelschule auf eine kommunale Bildungsmaßnahme im Vordergrund. Hasenjürgen stellt dazu die Perspektive des Schulleiters und die der verwiesenen Schüler_in dar. In ihrem macht- und institutionstheoretisch angelegten Vergleich pointiert sie etwa, dass Bezugnahmen auf Zuschreibungen fehlender Bildungsvoraussetzungen und Familienstrukturen mit Markierungen einer fehlenden Spielfähigkeit im schulischen Raum diese Verweisung argumentativ einleiten und nachfolgend begründbar machen.
Der Band ermöglicht mit der theoretisch-empirischen Verdichtung auf zentrale, vielschichtig aufbereiteter und zugleich fokussierter Diskursstränge gesellschaftskritischer Zugänge und der Zusammenführung dieser mit Kulturalisierungsprozessen im Bildungskontext wichtige empirische Einblicke in das hoch aktuelle Forschungsfeld. Er bietet ebenso Reflexionswissen für die pädagogische Arbeit, forschungspraktische Auseinandersetzungen und für die Lehre an. Die einzelnen Beiträge stechen dabei über ihre dem jeweiligen Gegenstand angemessene Einbettung - auch zum jeweiligen Forschungsstand - hervor und führen die Leser_innen in diese ein. Erwähnenswert ist dazu auch der Gastbeitrag von Putjata zu „Stimmen von eingewanderten Lehrkräften“ und von Schulze, die mit der Rahmung „angegriffene Identitäten“ eindringlich eine „Annäherung an das Bedrohliche antiziganistischer Stereotype“ vornimmt. Daher ist schließlich auch die Aufbereitung der Forschungsergebnisse und Auseinandersetzungen im Forschungsprozess als einzelne Beiträge besonders hervorzuheben.

Funding. Open Access funding provided by Projekt DEAL.

Open Access. Dieser Artikel wird unter der Creative Commons Namensnennung 4.0 International Lizenz veröffentlicht, welche die Nutzung, Vervielfältigung, Bearbeitung, Verbreitung und Wiedergabe in jeglichem Medium und Format erlaubt, sofern Sie den/die ursprünglichen Autor(en) und die Quelle ordnungsgemäß nennen, einen Link zur Creative Commons Lizenz beifügen und angeben, ob Änderungen vorgenommen wurden.

Die in diesem Artikel enthaltenen Bilder und sonstiges Drittmaterial unterliegen ebenfalls der genannten Creative Commons Lizenz, sofern sich aus der Abbildungslegende nichts anderes ergibt. Sofern das betreffende Material nicht unter der genannten Creative Commons Lizenz steht und die betreffende Handlung nicht nach gesetzlichen Vorschriften erlaubt ist, ist für die oben aufgeführten Weiterverwendungen des Materials die Einwilligung des jeweiligen Rechteinhabers einzuholen.

Weitere Details zur Lizenz entnehmen Sie bitte der Lizenzinformation auf http://creativecommons.org/licenses/by/4.0/deed.de 
Hier steht eine Anzeige.

\section{Springer}

